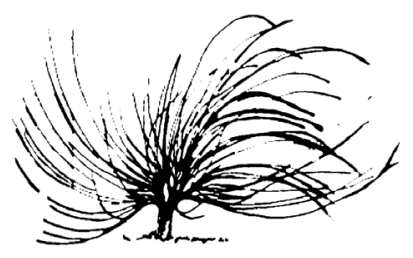

\title{
Modelo pedagógico helicoidal para la enseñanza y el aprendizaje de la química en entornos universitarios
}

\author{
Marcos Rojas Ulate ${ }^{1}$ \\ Universidad Nacional \\ Costa Rica \\ marcosrojasulate@gmail.com
}

\begin{abstract}
Resumen
La pedagogía científica universitaria posee una serie de deficiencias propias del sistema educativo que afectan los intereses y el crecimiento de sus estudiantes. Por tal motivo, se requiere un nuevo modelo pedagógico que proponga soluciones concretas a tales problemas. La pedagogía helicoidal establece la necesidad de una educación emocional, retroactiva, interconectada y en gradiente para que el estudiante no solo interiorice el conocimiento, sino que lo pueda poner en práctica durante cualquier momento de su vida académica y laboral. El conocimiento por medio de la pedagogía helicoidal puede ser creado de una manera más efectiva por medio del entendimiento y el estímulo de procesos mentales del ser humano en sus etapas tempranas y adultas. También es requerida la existencia de una plataforma sólida que facilite un proceso de aprendizaje helicoidal; lo anterior, aplicando acciones concretas como proyectos individualizados, cursos dinámicos con más de un profesor e incluso dejar de lado la clase magistral tradicional y la evaluación positivista. Desde luego que estos cambios no buscan un deterioro en la calidad ni rigidez de las carreras científicas, particularmente de la química; lo que se desea es mejorar la
\end{abstract}

http://dx.doi.org/10.15359/rep.esp-21.9

1 Químico Industrial. Máster, Pedagogía Universitaria en Universidad Nacional de Costa Rica. Estudiante de maestría en Biotecnología Molecular en Universidad de Barcelona. 
manera en la que las ciencias se enseñan y, además, demostrar al estudiante que su potencial es infinito si cambia su perspectiva y paradigma educativo respecto a su carrera profesional. Por tanto, el modelo helicoidal busca transformar la manera en la que se concibe la pedagogía universitaria en las ciencias exactas con fundamentos teóricos y propuestas praxeológicas concretas y aplicables en cualquier entorno académico mundial.

Palabras clave: Pedagogía, educación, química, conocimiento, ciencias exactas, modelo helicoidal.

\begin{abstract}
Scientific university pedagogy has several shortcomings related to the educational system that affect students' professional growth. Due to this situation, a new pedagogical model is required to solve these problems and limitations. Helical pedagogy establishes the necessity to set up an emotional, retroactive, interconnected, and gradient education to help students apply knowledge during every single moment of their labor or academic life. Through the stimulation of some mental processes related to human beings during their entire life (childhood or adulthood), knowledge can be created faster and in an easier way. Still, also a solid platform is required which contributes to the helical learning, for instance, individual projects, dynamic classes with two or more professors, and, of course, the avoidance of lectures and traditional evaluation methods. These changes do not mean that the quality of the educational system should be lower. The only objective of this new pedagogical method is to improve the way that chemistry is being learned by the students and also to make them more confident about their potential. This will happen if they change their perspective and vision about their studies and professional careers. This helical model seeks to transform the way to understand chemical pedagogy by using theoretical and praxeological proposals applicable in any context around the world.
\end{abstract}

Keywords: Pedagogy, education, chemistry, knowledge, science, helical model. 


\section{Introducción}

$\mathrm{L}$

as ciencias exactas son conocidas por la dificultad que representan para los estudiantes al avanzar a través de las distintas mallas curriculares. Las carreras pertenecientes al área de la química no son la excepción, por tal motivo, se propone un modelo pedagógico helicoidal para la enseñanza y el aprendizaje de la química en entornos universitarios. Tal propuesta debe ser entendida como una estructura helicoidal conformada por cuatro pilares fundamentales como lo son un aprendizaje en gradiente, interconectado, retroactivo que busque estimular emocionalmente y de manera constante a quien participa en este nuevo modelo pedagógico.

Desde la propia escuela, al estudiante se le estratifica según sus capacidades de aprendizaje; aquel alumno que es bueno para las ciencias exactas se le cataloga de inteligente y capaz, mientras que quien no muestra destrezas en dicha materia, se le ve como un alumno promedio y poco valioso para la sociedad. Así lo expresa Román (2012):

Se aprecia en América Latina un sistema de educación superior con un alto grado de desigualdad. En efecto, en la investigación realizada por Sverdlick, Ferrari, y Jaimovich, (2005) se sostiene que se trata de sistemas excluyentes desde el punto de vista social por sus propias características internas, que a su vez se asientan sobre otra exclusión. Es decir, se alude a un tipo de educación superior que selecciona a un conjunto de personas sobre un universo que ya pasó por fuertes procesos de selección social y educativa en períodos escolares previos. (p. 332)

Esta situación es generada, entre muchos otros factores, por la falta de una metodología de enseñanza didácticamente eficiente. Las ciencias exactas, específicamente la química, no pueden enseñarse y pretender que se aprenda de la misma manera que las ciencias económicas, políticas o lingüísticas. Cada área del conocimiento conlleva procesos de aprendizaje y técnicas didácticas distintas, situación ignorada por la gran mayoría de docentes en el mundo, quienes, sin mala intención, enseñan de igual forma un contenido científico a uno artístico o social.

En el ámbito de la educación superior, los fallos pedagógicos no solamente continúan, sino que se acrecientan. Toda aquella persona 
que haya pasado por un aula universitaria con profesores especializados en alguna ciencia exacta, probablemente ha experimentado muchísimas dificultades en su proceso de aprendizaje. Dificultades que van desde técnicas obsoletas para la transposición didáctica, docentes elitistas, desinteresados del alumno y con poco conocimiento del contexto empresarial; hasta exámenes pésimamente formulados y muchas más. Según lo detallan Fondón y Sarmiento (2010):

La realidad es que el nivel académico de los profesores en la universidad está garantizado dado el proceso de inserción en la carrera docente universitaria, pero esto mismo no ocurre en relación con la preparación para la función docente. Sin la necesaria formación específica para el desempeño docente, el profesor principiante puede quedar desprotegido y sentirse perdido ante el nuevo reto que afronta, dado que en esta etapa el profesorado se enfrenta a las diferencias entre los ideales y la realidad. (p. 22)

Por los motivos mencionados anteriormente, el surgimiento de este nuevo modelo pedagógico para la enseñanza y aprendizaje de la química en entornos universitarios resulta imperante. La necesidad de un cambio en el modelo actual justifica que profesionales en pedagogía con especialización en las ciencias exactas direccionen sus esfuerzos investigativos para encontrar una metodología que se ajuste tanto a las necesidades didácticas del entorno académico, así como al medio tecnológico, económico, industrial que la sociedad contemporánea demanda.

\section{Desarrollo}

A lo largo de muchos siglos, la educación se ha basado en un proceso vertical donde la persona docente domina las dimensiones físicas, éticas, técnicas y educativas, lo que quiere decir que quién enseña dirige a su conveniencia el proceso de enseñanza y aprendizaje desde una posición de poder y prácticamente de autorreconocimiento a su "gran sabiduría". Esta situación, sumada a la existencia de un modelo pedagógico centrado en el examen, principalmente en el resultado, han causado un gran deterioro en la educación científica nacional e internacional. Dicho en otras palabras, el método positivista aquí expuesto es para Pérez (2003) lo siguiente: 
En esta racionalidad, el positivismo se legitima como el paradigma válido y se instaura en la sociedad, a través de la concepción de cultura escolar sirviendo de base a la teoría del control social. La violencia a través de los símbolos, cercena a la crítica, y no hay apertura alguna al movimiento cualitativo de lo abstracto a lo concreto cuyo resultado conduce a una práctica teórica para producir lo concreto por vía del pensamiento. (p. 88)

Existe una cultura de examen, la cual hace que el curso y, por ende, los estudiantes estén en función de una prueba, generando a su vez que la población estudiantil aprenda sistemáticamente los conceptos, procesos y operaciones matemáticas para repetirlos sin mucho análisis. La materia estudiada, la forma en que se estudia, los problemas que se resuelven, están todos predeterminados por un formato evaluativo que tiene nula semejanza con la realidad que enfrentarán los grupos profesionales en ciencias exactas. Hace mucho tiempo que el examen perdió su dimensión pedagógica y se ha convertido en un vulgar filtro para cumplir requisitos mínimos de conocimiento mal almacenado.

El objetivo principal del examen es suprimir el error, irónicamente despreciar el error es la principal acción que hace perder credibilidad a este modelo evaluativo. El error como tal, es clave en todo proceso de aprendizaje. Este permite crecer y entender conceptos por medio del caos y la incertidumbre, pues exige la evolución y la interiorización del conocimiento en la persona estudiante; a tal grado que se sea capaz de solucionar la equivocación por medio de propuestas novedosas basadas en el ingenio y el autoanálisis.

El sistema educativo actual da por un hecho que, si la persona estudiante supera una serie de pruebas, este está capacitado para continuar a lo largo del extenso plan curricular de las carreras científicas. Se asume que en todo momento podrá retomar los conocimientos "ya aprendidos" para poderlos aplicar en otra circunstancia de su etapa universitaria. Por lo tanto, el examen tradicional es una herramienta propia del aprendizaje en gradiente que a continuación se explica, siendo ambos fenómenos complementarios e ideados para promover la memorización, restando así valor al aprovechamiento que el alumno o la alumna pueda dar al proceso de enseñanza y aprendizaje que experimenta.

A nivel universitario, el responsable directo de retener todo lo aprendido es el individuo estudiante, cuando en realidad esta tarea tiene 
que ser compartida. Fondón y Sarmiento (2010) se refieren a esta situación argumentando que:

La responsabilidad del docente no recae únicamente en la exposición de determinados contenidos, sino que es este quien debe facilitar todo el proceso de enseñanza y aprendizaje a través de una determinada metodología. Una buena preparación del contenido y de las estrategias para dirigir el aprendizaje puede facilitar mucho la consecución de los objetivos. (p. 23)

El modelo didáctico actualmente utilizado en las ciencias exactas se basa en un aprendizaje en gradiente y conductista. Descrito por Galagovsky (2007), la enseñanza de las ciencias exactas se basa en el método científico: "el método científico, desde otra visión pedagógica ingenua, consiste en ejecutar una secuencia de etapas. Esto supone una visión rígida y codificada de una metodología científica, cuyo correcto cumplimiento aseguraría resultados válidos, claros, lógicos y exactos" (p. 6). Este proceso de enseñanza y aprendizaje consiste en ir escalando el conocimiento, se empieza desde un nivel muy básico hasta que paulatinamente se incrementa tanto la cantidad de materia como el nivel de complejidad. La ineficiencia del modelo comienza cuando se da una ruptura en la continuidad de los conceptos. Navarro et al. (2014), hablan de cómo se corta la capacidad de aprender en el alumno cuando no comprende ciertos aprendizajes iniciales:

Partimos de la certeza de que las dificultades académicas no aparecen de repente, sino que se van gestando a lo largo de todo el proceso de enseñanza-aprendizaje. Desde los primeros pasos de un niño, empiezan a darse diferencias en el nivel de progreso de sus distintas capacidades y aunque en la mayoría de los casos los individuos compensan estos déficits aptitudinales durante su desarrollo, se observa un número elevado de casos en los que estas dificultades les impide adquirir, en un tiempo estimado como razonable, ciertos aprendizajes iniciales, fundamentales en la construcción del resto de conocimientos. (p. 74)

En ocasiones pueden pasar hasta dos o tres años en los cuales no se le pregunta a la persona estudiante ni se le reitera un tema en 
específico, pero de repente para poder entender un concepto avanzado necesita recordar una serie de definiciones previas. Como es lógico pensar, si la persona estudiante no recuerda o aprendió de mala manera la materia en el pasado (memorización en función de una evaluación escrita) o hasta se la explicaron deficientemente, tampoco entenderá lo que se le enseña en ese momento. Entonces, se irá arrastrando una serie de términos mal comprendidos que no le permitirán entender a fondo la materia estudiada en el presente y, por el contrario, acrecentará sus dudas e incomprensiones, pues recibe una gran cantidad de información en un tiempo muy corto.

Este sistema en gradiente no es del todo malo pues resulta necesario iniciar con conceptos sencillos para aprender poco a poco. La complejidad del conocimiento en este modelo se irá acrecentando conforme el estudiante avanza y adquiere mayores competencias para manejar circunstancias más complicadas. Lo que no puede ser válido es el querer justificar la funcionalidad del aprendizaje en gradiente por medio de la supuesta incapacidad de la persona estudiante de memorizar, cuando, en realidad, el choque cognitivo es desproporcionado producto de todos los conceptos nuevos que se pueden ver en una clase y todos los que son necesarios recordar en un mismo momento para poder avanzar en el aprendizaje.

El sistema conductista que se viene discutiendo, sin lugar a dudas, se basa en las necesidades de la sociedad y no de la persona, por tal motivo, según Pérez (2003):

La pedagogía que vendrá deberá ser un discurso para rescatar el sujeto del olvido, para reemprender la vigencia del sujeto en su mundo de vida, para ponerlo a pensar en el universo contenido en la totalidad y para redefinirse desde el uno dominado y para redefinir a la pedagogía desde sus bases ontológicas como discurso de la creatividad para impulsar la creatividad. (p. 91)

Es indispensable que el sistema de aprendizaje en gradiente cambie; no hay que desecharlo, pero sí resulta indispensable que evolucione por medio de la incorporación de un proceso en hélice o helicoide que, además, incorpore otras técnicas de enseñanza y estrategias de evaluación funcionales. Algunos ejemplos de éxito en la evolución de modelos 
pedagógicos, se presentan en Tünnerman (2008), en la siguiente cita se describe un ejemplo de la Universidad de Veracruz en México:

El modelo educativo de la Universidad Veracruzana parte de una redefinición del papel de la universidad y de su pertinencia social ante los cambios que se están dando en la sociedad contemporánea. En estas circunstancias, es urgente que los futuros profesionistas se desarrollen mediante nuevas formas de aprendizaje basadas en la educación integrada, es decir, que los estudiantes se formen con una visión inter y transdisciplinaria que les permita abordar la problemática de su disciplina. (p. 47)

\section{Modelo pedagógico helicoidal}

Para comprender los alcances de este nuevo modelo helicoidal para la enseñanza de la química, es necesario pensar, en primera instancia, en el significado matemático de espiral. Se debe imaginar una línea curva que se va alejando progresivamente de un punto concéntrico a la vez que gira alrededor de él en múltiples ocasiones.

El aprendizaje en espiral es un proceso de crecimiento continuo donde el conocimiento interactúa una y otra vez con varias etapas previas para su evolución. Es decir, desde este punto de vista, el conocimiento se retoma constantemente para ser evaluado y mejorado, así, lo aprendido nunca se desecha ni olvida, sino que se utiliza de base para lo siguiente. Sin embargo, esta base es reconstruida siempre mediante este proceso dinámico, nunca se dará por finalizada o terminada.

El proceso de aprendizaje en espiral es muy eficiente, no obstante, se mueve y crece en un plano horizontal dejando de lado la posibilidad de expandirse tridimensionalmente para alcanzar horizontes más amplios y distantes. Una hélice cónica, en cambio, siempre es tridimensional: se trata de una línea curva continua, con pendiente variable y no nula que gira alrededor de un cilindro y que se va abriendo cada vez más respecto al punto de origen. Este movimiento helicoidal cónico funciona para ilustrar lo que se pretende mediante esta metodología de enseñanza y aprendizaje que dinamiza la educación dejando de ser estática como ocurre en un proceso de gradiente. Consecuentemente, una pedagogía helicoidal permite un avance gradual no cíclico, pero sí retoma lo aprendido previamente desde varios ángulos (distintos cursos 
o módulos pasados). Con esta metodología, se busca ir expandiendo los conceptos a través de la interconectividad que estos puedan tener a razón de las distintas perspectivas que necesita cada nuevo curso en particular. En palabras simples, se desea recordar y conectar al mismo tiempo la mayor cantidad de conocimiento ya verdaderamente interiorizado con el nuevo por aprender; para que, independientemente del enfoque que se vaya a trabajar, el conocimiento nunca sea mal comprendido ni olvidado.

Ahora bien, ¿cómo lograr que el conocimiento sea constantemente utilizado por la población estudiantil y que se encuentre interconectado a lo largo del plan de estudios en una carrera de ciencias exactas?

\section{Estrategias pedagógicas para alcanzar el modelo helicoidal}

Fomentar un cambio curricular y didáctico son las dos principales acciones para incentivar esta metodología de trabajo, se requieren ejes temáticos más amplios y diversos, no estratificar tanto la materia, sino verla como una sola unidad de conocimiento. Martínez et al. (2012) concuerdan en la necesidad de planificar la malla curricular en función del estudiantado y no del beneficio de la universidad:

Para ello es necesaria una transformación profunda de algunos aspectos de la planificación y el desarrollo de los planes de estudio universitarios. Si no se realiza esa transformación es muy posible que la implementación de estos currículos no conduzca a cambios reales en los egresados $\mathrm{y}$, por tanto, se perderán las ventajas que ofrece formar a los futuros profesionales. (p. 90)

Los mismos conceptos que se estudian en una materia de la malla curricular, se llegan a trabajar en otra, por lo cual es requerido utilizar esas relaciones para que la persona estudiante no sienta que está recibiendo información vieja, sino que el conocimiento tiene múltiples aplicaciones y alcances. Indudablemente, se necesita una mayor hibridación del sistema educativo científico con el fin de que se comporte como una organización viva, interrelacionada, complementaria. Es decir, comunicándose constantemente de tal forma que el conocimiento nuevo llegue inmediatamente a toda la comunidad académica por medio de artículos científicos, investigaciones de campo, literatura analítica y explicativa o por medio de exposiciones o ponencias en tiempo real. 
También se requieren técnicas de enseñanza más integrales que mantengan latentes los conceptos en el alumnado. Desechar, aunque sea parcialmente el modelo positivista actual de clases magistrales y evaluaciones escritas es imperante. Sánchez et al. (2013) así lo demuestran dado los cambios tecnológicos que influyen al estudiantado:

Profesores con experiencia en implementación de clases magistrales, mencionan una queja generalizada sobre la incapacidad de los estudiantes en mantener la atención en ellas por más de 20 minutos, e inclusive Gildroy [11], considera como posible explicación de este hecho que los jóvenes de hoy en día están acostumbrados al procesamiento veloz de gran cantidad de información a partir de pantallas de televisores y computadores, por lo que encuentran dificultad para mantener la atención al ritmo "lento" de una clase magistral. (p. 18)

La pedagogía helicoidal busca que exista un principio o una base, no obstante, nunca buscará un final, sino que fomentará el aprendizaje para toda la vida, evitando que se vea como una simple etapa; este enunciado es un principio básico de esta metodología, cuyo centro de acción siempre será el estudiante.

El modelo helicoidal toma de base las ideas constructivistas que, de acuerdo con lo expuesto por Ortiz (2015), no deben verse como la acción pedagógica de dejar en libertad a la población estudiantil para que aprendan a su propio ritmo, sino que deben ser entendidas de la siguiente manera:

Esta es una concepción errónea del constructivismo puesto que este enfoque, lo que plantea en realidad es que existe una interacción entre el docente y los estudiantes, un intercambio dialéctico entre los conocimientos del docente y los del estudiante, de tal forma que se pueda llegar a una síntesis productiva para ambos y, en consecuencia, que los contenidos son revisados para lograr un aprendizaje significativo. (p. 94)

Tal como se puede inferir en dicha cita, gran parte del personal docente entiende por constructivismo el hecho de dar el liderazgo de la clase al estudiantado. Sin embargo, con esta corriente pedagógica 
lo que realmente se pretende es fomentar en la población estudiantil la creación de un conocimiento propio bajo la guía del profesor o la profesora. De esta forma, se entiende que el ser humano percibe, interpreta y organiza la información que recibe de su entorno de forma distinta y que dicha individualidad es necesario explorarla y fomentarla de la mejor forma posible.

Por tal motivo, con el modelo helicoidal se busca rescatar el individualismo existente en el proceso de aprendizaje para respetar los procesos mentales en cada estudiante. No se desea utilizar métodos de enseñanza y evaluación estandarizados que no contribuyen a la creación de conocimiento, sino que establecen barreras para muchos de los estudiantes de las ciencias exactas y naturales.

Para convertir a la persona estudiante en responsable de su propio saber, se puede implementar el aprendizaje por proyectos que trae consigo enormes beneficios pedagógicos, lo cuales se analizan a continuación. De acuerdo con Blank (1997, según se cita en Estrada, 2012), el aprendizaje por proyectos se define como:

Bajo la concepción constructivista, el aprendizaje por proyectos se vislumbra como un escenario ideal, donde los alumnos se responsabilizan de su propio aprendizaje, ya que esta estrategia de enseñanza establece un modelo de instrucción genuino en el que los estudiantes son capaces de planear, implementar y evaluar proyectos que tienen aplicación en el mundo real. (p. 128)

Con el método de enseñanza helicoidal se pretende dar completo protagonismo a la persona estudiante en el desarrollo de sistemas de trabajo e ideas innovadoras. El hecho de desarrollar sus propios proyectos, le permitirá ir entendiendo realmente los conceptos teóricos a la vez que puede observar la aplicación de los mismos en el mundo real, al menos a una pequeña escala. Al mismo tiempo, se obliga a retomar conceptos aprendidos previamente y se motiva dado que el proyecto será de su completa elección.

Otra metodología de enseñanza, similar a la planteada anteriormente, que en definitiva estimula la pedagogía helicoidal, es el aprendizaje basado en retos; así, en Moore (2013), se señala: 
El aprendizaje basado en retos tiene sus raíces en el aprendizaje vivencial, el cual tiene como principio fundamental que los estudiantes aprenden mejor cuando participan de forma activa en experiencias abiertas de aprendizaje, que cuando participan de manera pasiva en actividades estructuradas. En este sentido, el aprendizaje vivencial ofrece oportunidades a los estudiantes de aplicar lo que aprenden en situaciones reales donde se enfrentan a problemas, descubren por ellos mismos, prueban soluciones e interactúan con otros estudiantes dentro de un determinado contexto (For interns, experience isn't always the best teacher, párr. 3-5).

Generalmente se tiende a confundir el aprendizaje por proyectos y el aprendizaje basado en retos, no obstante, de acuerdo al planteamiento de Gaskins et al. (2015):

El aprendizaje basado en retos comparte características con el aprendizaje basado en proyectos. Ambos acercamientos involucran a los estudiantes en problemas del mundo real y los hacen partícipes del desarrollo de soluciones específicas. Sin embargo, estas estrategias difieren en que en lugar de presentar a los estudiantes un problema a resolver, el aprendizaje basado en retos ofrece problemáticas abiertas y generales sobre las cuales los estudiantes determinarán el reto que abordarán. (p. 35)

En múltiples centros universitarios alrededor del mundo, como en el Tecnológico de Monterrey, se establece la importancia de hacer consciente a la población estudiantil del proceso de aprendizaje que utiliza para que pueda sacar mayor provecho a la metodología y principalmente al método didáctico del cual es partícipe:

La implementación del aprendizaje basado en retos y proyectos también debe incorporar elementos metacognitivos en su evaluación, que permitan al alumno reflexionar sobre los aprendizajes logrados o no durante el proceso. El ser consciente de las lecciones aprendidas, de los conocimientos adquiridos durante la puesta en marcha de la solución y de las habilidades transversales como el trabajo en equipo, la comunicación efectiva o el uso eficiente de la tecnología, es particularmente relevante en este 
acercamiento. (Observatorio de Innovación Educativa del Tecnológico de Monterrey, 2015, p. 13)

Tanto el aprendizaje por proyectos, como el aprendizaje basado en retos, representan una serie de dificultades ya sea para implementar el modelo pedagógico helicoidal o cualquier otro formato de aprendizaje. Algunas situaciones complicadas a presentarse pueden ser: una difícil transición para la comunidad educativa, pues tanto alumnos, profesores y administrativos tienen que adaptarse a un cambio de paradigma. También se requiere de mayor tiempo y esfuerzo por parte la población estudiantil y el personal docente, así como una modificación curricular por parte de la institución educativa que integre este tipo de modelos de aprendizaje. Para Poot-Delgado (2013):

Los profesores pueden carecer de la habilidad de facilitar. La mayor parte de los profesores no tienen la capacitación necesaria para trabajar con los grupos de alumnos. La inercia en continuar siendo el centro de la clase y exponer información es muy fuerte. El área de mayor dificultad para los profesores se observa en un deficiente dominio de los fenómenos de interacción grupal (cohesión, comunicación, competencia, etc.). (p. 313)

\section{Teorías de aprendizaje para fundamentar el método helicoidal}

Un recurso pedagógico a considerar para su aplicación en esta nueva metodología helicoidal y, además, una de las principales razones por las cuales se plantea su utilización, son los procesos mentales de aprendizaje en el interior de cada individuo. El ser humano tiene la capacidad de analizar constantemente su entorno, sus errores y aciertos, incluso de interpretar y principalmente aprender de sus acciones pasadas.

Para Jaramillo y Simbaña (2014), la metacognición puede entenderse a partir de su etimología: "si descomponemos etimológicamente la palabra metacognición, tenemos que meta, proviene del prefijo griego que significa "más allá" y cognición del latín cognoscere que significa "conocer". La metacognición, en general se entiende como pensar sobre el pensamiento" (p. 300).

El sistema de aprendizaje actual, fomenta la repetición de conceptos y habilidades, por lo tanto, no incentiva el autoconocimiento del individuo sino una reproducción simplista por nuestros procesos 
mentales. Por este motivo, el modelo conductista no realiza un aporte significativo al desarrollo del conocimiento, por otro lado, el modelo helicoidal busca un cuestionamiento continuo en el interior del individuo por medio del caos y la incertidumbre. Si todo se le facilitara a la población estudiantil, no se le estaría ayudando, pues se estaría entorpeciendo su capacidad de adaptación a su entorno y en el desarrollo de su condición de ser pensante.

Para Capilla (2016), es necesario la reiteración y la interacción de los conceptos ya adquiridos para poder incorporar los nuevos, lo cual confirma uno de los pilares de esta metodología como lo es el aprendizaje retroactivo:

La estructura cognitiva, representada por las ideas o conceptos existentes, funciona como anclaje para establecer la conexión significativa con nuevos conceptos. Empero, este proceso no es unidireccional sino bidireccional dado que tanto los conceptos o ideas preexistentes como los nuevos que se integran evolucionan conformando una nueva estructura. Es decir, cuando se concreta la conexión lógica y sustancial entre un concepto prexistente en la estructura cognitiva y una nueva información o concepto presentado, este segundo adquiere significado, lo que favorece que ambos evolucionen. (p. 52)

A continuación, un análisis del proceso de aprendizaje de fracciones matemáticas realizado por Capilla (2016) que ejemplifica el Principio de Asimilación de Ausubel:

La noción o concepto de fracción presente en la estructura cognitiva del alumno servirá de subsunsor para la aprehensión significativa del concepto de fracción equivalente. Esta, a su vez, permitirá comprender que una fracción puede representarse simbólicamentecon diferentes dígitos. En este mismo sentido, la adición de números naturales será el subsunsor para el concepto de adición de fracciones comunes, y este segundo concepto permite comprender que la adición no se limita a los números naturales, se extiende a las fracciones comunes. (p. 52) 
Por medio de este ejemplo, se puede concluir que, a partir de un conocimiento previo, el cerebro utiliza tal información para usarla como punto de partida para retener nuevos datos con características similares. El modelo pedagógico helicoidal, se basa en esta forma de funcionamiento del cerebro para reutilizar conocimientos previos y, así, usarlos como plataforma para adquirir más información que posiblemente evolucione en conocimientos más complejos e interconectados que los pre existentes.

Una idea muy similar que también es aplicable a las intenciones del método helicoidal que, de igual manera, es planteada en Ausubel et al. (2009, según se citan en Capilla, 2016), es el aprendizaje significativo:

El aprendizaje significativo ocurre cuando nuevas ideas, conceptos y proposiciones pueden ser aprendidos de manera significativa en la medida en que otros conceptos, ideas o proposiciones relevantes estén claros y disponibles en la estructura cognitiva del individuo. De esta manera el sujeto establece una relación sustantiva entre los conceptos que posee y la nueva información. El nuevo objeto de aprehensión debe ser relacionable con la información preexistente en el sujeto, es decir, debe cumplir con el criterio de relacionalidad. En síntesis, el aprendizaje significativo es la adición de subsumidores o conocimiento previo, estructura cognitiva e interés o disposición. (p. 52)

Por último, para cerrar el análisis del aprendizaje significativo que es una de las bases de la fundamentación pedagógica en el modelo helicoidal, resulta necesario citar a Viera (2003), quien describe claramente las rutas de aprendizaje que conforman el aprendizaje significativo y que, por ende, deben ser tomadas en cuenta para el éxito de este nuevo modelo de enseñanza:

Ausubel distingue tres formas de aprendizaje significativo. El aprendizaje representacional: tipo básico de aprendizaje significativo. En él se asignan significados a determinados símbolos (palabras), se identifican los símbolos con sus referentes (objetos, eventos, conceptos). Aprendizaje de conceptos: los conceptos representan regularidades de eventos u objetos, y son representados también por símbolos particulares o categorías y 
representan abstracciones de atributos esenciales de los referentes. Por último, el aprendizaje proposicional: la tarea no es aprender significativamente lo que representan las palabras aisladas o combinadas sino significados de ella como un todo. (p. 38)

El modelo pedagógico helicoidal para la enseñanza y el aprendizaje de la química en entornos universitarios, busca que cada quien aprenda y retenga la información de la forma que mejor le parezca. Por este motivo, se incentiva a la población estudiantil a ser libre de aprender de la forma en la que más disfrute y como mejor se sienta, entienda y retenga el conocimiento; es decir, es la persona estudiante la que decide el tipo de inteligencia que desea utilizar para aprender.

A razón de clarificar el tema de inteligencias múltiples y resaltar la necesidad de que cada estudiante decida cómo quiere aprender, vale la pena citar a Suárez et al. (2010), quienes buscan reconocer diferentes tipos de inteligencias:

Por otra parte, reconocer la existencia de inteligencias diversas, supone considerar recursos diferentes para cada estilo de aprendizaje. Así, se postula que el contenido puede presentarse a partir de cinco modalidades diferentes que responden a las diferentes tipologías de la inteligencia, de manera que podrían concebirse como varias puertas de acceso al conocimiento. Éstos son: el narrativo, que utiliza la narración como soporte del concepto que se desea enseñar y podría identificarse a la inteligencia lingüística; el lógico-cuantitativo que utiliza consideraciones numéricas o razonamientos deductivos y se asocia a la inteligencia lógico-matemática; el fundacional; referido a interrogantes de tipo filosóficos que refiere quizá a la inteligencia intrapersonal y/o interpersonal; el estético, orientando a los aspectos sensoriales, implicando a la inteligencia musical y a la espacial; $y$, finalmente, el experimental que orientada, entre otras cosas, hacia actividades de manuales, podría llegar a vincularse a la inteligencia cinético corporal. (p. 88)

Ligado al tema de las inteligencias múltiples, el modelo helicoidal también pretende rescatar ciertas formas de aprendizaje presentes en la niñez. Si se considera la facilidad con la que los niños y niñas llegan a retener cantidades incalculables de información, es de esperarse que 
se generen cuestionamientos acerca del porqué de tal habilidad. Para explicar este fenómeno, es necesario entender los procesos de aprendizaje que atraviesan los infantes. Sin entrar en muchos tecnicismos, es posible decir que cualquier persona es capaz de entender que los niños y niñas aprenden jugando, de manera natural. Sin estar conscientes en que están creando conocimiento, los seres humanos en etapas tempranas, sin una intervención por parte de un modelo pedagógico, son capaces de entender muchas circunstancias de la vida cotidiana sin necesidad de explicación previa. Precisamente por estas capacidades cognitivas, innatas en toda persona, el modelo helicoidal apela para fomentar un aprendizaje libre sin rutas prestablecidas ni paradigmas impuestos.

En la teoría constructivista planteada por Jean Piaget, se busca explicar de manera detallada el proceso de enseñanza y aprendizaje que experimenta todo ser humano. Las etapas iniciales en los procesos de aprendizaje son las que más interesa entender para el desarrollo de la propuesta de enseñanza helicoidal. Es en tales instancias donde el aprendizaje es más intuitivo y emocional, ambas características son los recursos que desea rescatar el modelo, pues son donde el aprendizaje significativo se potencia y se llega a consolidar a lo largo de toda la vida del individuo. En su artículo, Saldarriaga et al. (2016) describen detalladamente las etapas tempranas de desarrollo cognitivo planteadas por Piaget:

La primera de ellas (2 a 7 años), es identificada por el autor como preoperatoria, se presenta con el surgimiento de la función simbólica en la cual el niño, comienza a hacer uso de pensamientos sobre hechos $u$ objetos no perceptibles en ese momento. La inteligencia o razonamiento es de tipo intuitivo ya que no poseen en este momento capacidad lógica. Los niños son capaces de utilizar diversos esquemas representativos como el lenguaje, el juego simbólico, la imaginación y el dibujo. Se caracteriza por la presencia de varias tendencias en el contenido del pensamiento: animismo, realismo y artificialismo. ya que suelen atribuir vida y características subjetivas a objetos inanimados, pues comprenden la realidad a parte de los esquemas mentales que poseen. (p. 132)

Como queda evidenciado, los primeros años de vida poseen un carácter más intuitivo y no esquematizado. Es necesario que, tanto en la enseñanza de las ciencias exactas como en otras áreas, se explote el 
juego simbólico, animismo, representación simbólica e inteligencia y razonamiento intuitivo. Con tal práctica, se quiere volver a los orígenes de la creación del conocimiento en el ser humano, pero contextualizado $\mathrm{y}$ adaptado para un ser humano cognitivamente desarrollado propio de una etapa universitaria.

Las emociones son otro factor fundamental en el proceso de aprendizaje; si estas no son incluidas durante el estudio, la cognición del estudiante no será igual de efectiva. Primeramente, es importante definir qué es la emoción, ante lo cual García (2012) propone:

Esto significa que, las emociones son eventos o fenómenos de carácter biológico y cognitivo, que tienen sentido en términos sociales. Se pueden clasificar en positivas cuando van acompañadas de sentimientos placenteros y significan que la situación es beneficiosa, como lo son la felicidad y el amor; negativas cuando van acompañadas de sentimientos desagradables y se percibe la situación como una amenaza, entre las que se encuentran el miedo, la ansiedad, la ira, hostilidad, la tristeza, el asco, o neutras cuando no van acompañadas de ningún sentimiento, entre las que se encuentra la esperanza y la sorpresa. (p. 3)

Para el método pedagógico helicoidal resulta fundamental que la persona estudiante se sienta identificada emocionalmente con lo que se estudia. Cuando un individuo involucra sentimientos positivos en el emprendimiento de una tarea, los resultados siempre serán mucho más valiosos para ella. Dentro de este proceso de aprendizaje emocional, existen una acción que debe incluirse para alcanzar una didáctica exitosa y funcional; la percepción, que Ortiz (2009) postula de la siguiente manera:

La percepción es el conocimiento de las respuestas sensoriales a los estímulos que las excitan. Por la percepción se distinguen y diferencian unas cosas de otras, el ser del mundo, la realidad de las otras cosas. Las sensaciones son una condición necesaria, pero no suficiente de la percepción sensible. (p. 16)

La mejor manera de fijar la atención de una persona en alguna acción, evento o contexto determinado, es por medio de las emociones. $\mathrm{Si}$ el individuo se involucra emocionalmente es mucho más difícil que 
olvide lo acontecido y, entonces, podrá recordar esa información adquirida a lo largo de su vida. Para entender mejor los procesos que suceden en el cerebro para asimilar los datos que retiene, es necesario la utilización de la neurociencia para poder describirlos.

Es lógico pensar que las emociones están directamente asociadas a la memoria, porque los acontecimientos mejor recordados por el cerebro son aquellos que poseen una alta significancia en la vida del ser humano. En su columna científica, Manés (2016) explica acertadamente, desde el campo de la neurología, la manera en que los recuerdos se reactivan para poderlos utilizar:

Décadas de investigación científica han establecido que la consolidación de la memoria a largo plazo exige la síntesis de proteínas en los caminos neuronales del cerebro, pero nadie sabía que también hacía falta una síntesis de proteínas después de recuperar un recuerdo para poder transportarlo del punto donde está almacenado hasta el lugar que es interpretado por el cerebro, lo que implica también que se está consolidando en ese momento. Esto resultó una excelente pista bioquímica de que, al menos, algunos tipos de recuerdos hay que reescribirlos neuronalmente cada vez que se recuperan. Por eso al evocar una memoria, recuerdo o información en el cerebro, la estamos recreando y así tenemos menor precisión del recuerdo original. (p. 1)

Aunque suene contradictorio, la ciencia muestra que, si uno tiene una memoria, un dato o un concepto, cuanto más se usa, más se modifica. Por lo anterior, principalmente a la hora de recordar datos científicos muy específicos, es crucial estar estimulando los conocimientos para que la ruta cerebral que los define no cambie tanto en relación a la información original, es decir, estimular la retroactividad de los datos aprendidos.

Por medio de las teorías de aprendizaje y recursos pedagógicos descritos anteriormente, se puede confirmar la necesidad de una propuesta pedagógica que involucre las emociones, la retroactividad del conocimiento, la interconectividad y que se presente en gradiente con la finalidad de que resulte realmente efectiva para la persona estudiante. 


\section{Propuestas del modelo pedagógico helicoidal}

Para poder plasmar las ideas descritas a lo largo del ensayo, se plantea una serie de acciones concretas desde el punto de vista pedagógico y didáctico con el afán de otorgarle funcionalidad al sistema helicoidal en cuestión:

1. Como primera propuesta, es necesario dinamizar el formato de clase magistral, actualmente utilizado por los y las docentes de química. Para tal objetivo, se puede incorporar ejemplos integrados, situaciones reales para la contextualización de los conceptos y actividades de aprendizaje diversas; también es necesario hacer partícipe a la persona estudiante a lo largo de la sesión para que la clase magistral no se convierta en un monólogo por parte del personal docente. Como parte de este rescate de los aspectos positivos del sistema positivista actualmente utilizado, se puede mantener exámenes escritos. Sin embargo, también es necesario utilizar otros recursos, como, por ejemplo, entrevistas. En el caso de las investigaciones sociológicas, particularmente en los posgrados, aunque cada vez más frecuente en niveles de grado, suelen mostrar mucho más predominio las opciones cualitativas, destacándose la entrevista como un tipo de técnica de investigación dominante (Robert y Lisdero, 2016). En el ámbito de la evaluación científica, se puede realizar entrevistas individuales con demostraciones teóricas por parte del alumno como sistema de evaluación alternativa.

2. Es indispensable fomentar un modelo de aprendizaje basado en proyectos por investigación científica y retos reales. Siempre es necesario la impartición de conceptos teóricos, pero no se debe caer en el adoctrinamiento de la información, ni extensión de los conceptos, sino fomentar la aplicación de los mismos. Como parte de esta propuesta de aprendizaje por proyectos, es necesario fomentar la memoria visual y no solo la escrita y auditiva. Hay que diversificar el aprendizaje para que la población estudiantil pueda elegir la forma en la que desarrollará sus proyectos, bajo el principio de las inteligencias múltiples.

3. La correcta utilización del espacio físico también es un factor fundamental para el buen funcionamiento de la población estudiantil. Crear espacios abiertos de trabajo común donde alumnos y alumnas 
con mayor experiencia ayuden a los más jóvenes con la elaboración de sus trabajos, iniciativa muy utilizada en centros de estudio europeos.

4. Otra propuesta es la existencia de una malla curricular integrada e interactiva donde las materias se encuentren entrelazadas y donde exista una repetición consciente e intuitiva de conceptos previos para que estos vayan evolucionando en la medida que se aprueban los cursos. El planteamiento anterior es uno de los ejes principales del modelo helicoidal porque permite al estudiante mantener sus conocimientos frescos para aplicarlos durante cualquier circunstancia.

5. Bajo la misma línea de trabajo del diseño curricular, se propone no estructurar las materias sino establecerlas como módulos. Al igual que se realiza en otros campos de estudio como las ciencias sociales, se pueden llevar clases en paralelo que introduzcan temas interconectados con varios profesores a la vez que se intercambien según el tema de su especialidad. El currículo moderno lo definen Avendaño-Castro y Parada-Trujillo (2013):

El currículo es un conjunto de procesos de formación porque implica la transversalidad de los saberes en situaciones concretas, además que busca un aprendizaje de tipo integrador que permita cambios relevantes y significativos en el sujeto que aprende. Con ello se aísla la idea del trabajo por áreas y asignaturas, la clase magistral (en torno al maestro) y el direccionamiento vertical de la enseñanza. (p. 164)

6. Cambiar la escala de evaluación. No resulta conveniente que en la enseñanza de las ciencias siga prevaleciendo un modelo de notas que no demuestran ciertamente el aprendizaje de la población estudiantil. Por consiguiente, dicha práctica tiene que ser erradicada para, en su lugar, darle espacio al trabajo y estudio por objetivos, las evaluaciones tienen que ser una retroalimentación no una simple calificación resultadista.

7. Disminuir contenidos teóricos. Una idea predeterminada para las ciencias exactas, es la cantidad de materia inverosímil que debe abarcarse para que se considere una enseñanza de calidad. En realidad, esta práctica didáctica es completamente absurda; en su lugar se debería dar una regulación apropiada de la carga académica. En caso de ser necesario, debido a la tecnicidad de los contenidos, se 
puede extender el tiempo de graduación de la población estudiantil en un sistema cuya idea sea producir profesionales excelentes no de forma rápida como si de una producción en masa se tratara.

8. Uno de los puntos en los que la química se encuentra más rezagada es en la introducción de tecnología en sus aulas. El uso de la tecnología y plataformas académicas digitales, así como la producción digital por parte de los estudiantes, es una obligación para cualquier unidad académica de ciencias exactas. En un mundo cada vez más digitalizado, la utilización de herramientas pedagógicas virtuales, facilita enormemente la tarea de enseñanza a los profesores, al mismo tiempo que dinamiza el aprendizaje, volviéndolo más interesante para quien recibe la información.

9. Como penúltimo punto a plantear, se encuentra el fomento de la consolidación del conocimiento que debe ser creado en la propia unidad académica. Esto por medio de una editorial para libros o videos realizados por las personas estudiantes e, incluso, por los y las docentes. Hacer una videoteca con los proyectos realizados estimulará enormemente a la población estudiantil, pues se observará como los trabajos son tomados en cuenta como material de apoyo para futuras generaciones.

10. La gestión y política académica son responsables directos del éxito de la población estudiantil, tanto fuera como dentro de las aulas. En la mayoría de facultades de química del mundo, hace falta integrar la industria con la academia. Siempre ha sido tema de discusión entre ambas partes la protección de la información y los derechos de autor; los catedráticos tienen que abrir su perspectiva y entender que es posible vincular la investigación académica con la industrial, estableciendo límites claros y sin permitir ningún tipo de injerencia tanto de un lado como del otro. Estimular el trabajo cooperativo entre laboratorios universitarios y privados a través de convenios para que los y las estudiantes trabajen en investigaciones verdaderas de forma obligatoria, será muy beneficioso para su crecimiento profesional. Para Castillo-Cedeño et al. (2015), resulta esencial gestionar un entorno universitario significativo, con experiencias enriquecedoras para los y las estudiantes

Las innovaciones se caracterizan por políticas claras de motivación que cautivan a la totalidad de miembros de la comunidad 
educativa, quienes comprenden y se identifican plenamente con el compromiso de gestar experiencias de aprendizaje y de vida que revelen y atiendan el bienestar común. (p. 6)

\section{Reflexión final}

Existen gran cantidad de ejemplos de éxito donde propuestas de aprendizaje innovadoras se han llevado a cabo. Por esta razón, no se debería dar espacio al miedo a la hora de generar cambios en la pedagogía universitaria de cualquiera que sea la facultad de ciencias exactas.

Algunos países que han revolucionado sus métodos pedagógicos de enseñanza son: Finlandia, donde no existen las tareas ni exámenes y, además, la educación es completamente pública, Argentina y Chile, líderes latinoamericanos en cuanto a propuestas de educación se refiere, sobre todo en centros de estudio como la Universidad de Córdoba y la Universidad de Chile; además, universidades en Dinamarca, donde se comienzan a aplicar los ambientes de aprendizaje abiertos donde convergen estudiantes de todos los niveles para interactuar mutuamente, dicha idea fue tomada en cuenta para una de las propuestas didácticas descritas anteriormente. La Universidad de Sussex en Inglaterra, es otro ejemplo a seguir en actividades alternativas de aprendizajes en contextos de mundo real; con este tipo de actividades se busca insertar a los y las estudiantes a situaciones reales con un contexto verdadero bajo la premisa que el mayor conocimiento se encuentra en las calles.

Propuestas concretas como las planteadas anteriormente son claros ejemplos del panorama hacia donde el diseño curricular, la didáctica, la epistemología y la pedagogía como tal deben de dirigirse. Este cambio resulta necesario para el mejoramiento de las condiciones actuales donde se llevan a cabo los procesos de enseñanza y aprendizaje.

De esta forma, demostrar a la persona estudiante que su potencial es infinito, cambiar su perspectiva y su paradigma respecto a su carrera profesional, tienen que ser objetivos primordiales de toda metodología de enseñanza universitaria. El modelo pedagógico helicoidal para la enseñanza y aprendizaje de la química busca transformar la manera en la que se imparten las lecciones en las aulas, no obstante, esto solamente resulta ser una pequeña parte de la razón de la propuesta.

Este cambio de perspectiva pretende interiorizar en los alumnos la importancia de crear un futuro próspero, pero principalmente la idea 
de estudiar con disfrute del proceso. La única manera de ser realmente exitoso es por medio del trabajo duro, el compromiso y el amor por lo que se hace, lo cual, a su vez, se consigue con una educación que estimule continuamente a la persona estudiante y lo transporte a lo largo del proceso de enseñanza a lugares que parecen lejanos incluso para la imaginación y las emociones. No se busca menospreciar el trabajo ni la relevancia de los y las docentes, ni demás personal universitario, sino que se propone encontrar un balance por medio del trabajo colaborativo entre ambas partes. Ante esto, no se puede olvidar bajo ninguna circunstancia que la persona estudiante es eje de la plataforma educativa y, por tanto, debe tener el control de las decisiones que involucren cómo se procederá en la creación del conocimiento.

Con la propuesta pedagógica helicoidal no se busca alejar a la persona estudiante de la rigurosidad y el enorme esfuerzo que conlleva estudiar una carrera como química. Sencillamente, este planteamiento pretende humanizar las mal llamadas "ciencias duras" para que, por medio de una educación emocional, retroactiva, interconectada y en gradiente, el o la estudiante alcance un estado de plenitud racional y emocional durante su proceso de aprendizaje.

\section{Referencias}

Avendaño-Castro, W. y Parada-Trujillo, A. (2013). El currículo en la sociedad del conocimiento. Educación y Educadores, 16(1), 159174. https://www.redalyc.org/pdf/834/83428614008.pdf

Capilla, R. (2016). Habilidades cognitivas y aprendizaje significativo de la adición y sustracción de fracciones comunes. Cuadernos de Investigación Educativa, 7(2), 49-62. http://www.redalyc.org/ pdf/4436/443649571004.pdf

Castillo-Cedeño, I., Flores-Davis, L. y Miranda-Cervantes, G. (2015). Gestión académica saludable en el contexto universitario. Revista Electrónica Educare, 19(3), 1-25. http://dx.doi.org/10.15359/ree.19-3.24

Estrada, A. (2012). El aprendizaje por proyectos y el trabajo colaborativo, como herramientas de aprendizaje, en la construcción del proceso educativo, de la Unidad de aprendizaje TIC'S. RIDE Revista Iberoamericana para la investigación y el Desarrollo Educativo, 3(5), 123-138. http://www.redalyc.org/pdf/4981/498150313010.pdf

Fondón, M. y Sarmiento, A. (2010). Principales problemas de los profesores principiantes en la enseñanza universitaria. 
Formación Universitaria, 3(2), 21-28. http://dx.doi.org/10.4067/ S0718-50062010000200004

Galagovsky, L. (2007). Enseñar química vs. Aprender química: una ecuación que no está balanceada. Química Viva, 6(Sup), 0. https://www.redalyc.org/pdf/863/86309909.pdf

García, J. (2012). La educación emocional, su importancia en el proceso de aprendizaje. Revista Educación, 36(1), 1-24. http://www. redalyc.org/pdf/440/44023984007.pdf

Gaskins, W., Johnson, J., Maltbie, C. y Kukreti, A. (2015). Changing the Learning Environment in the College of Engineering and Applied Science Using Challenge Based Learning. International Journal of Engineering Pedagogy, 5(1), 33-41. http://dx.doi. org/10.3991/ijep.v5i1.4138

Jaramillo, L. y Simbaña, V. (2014). La metacognición y su aplicación en herramientas virtuales desde la práctica docente. Sophia, Colección de Filosofia de la Educación, 16, 299-313. http://www. redalyc.org/pdf/4418/441846097014.pdf

Manes, F. (2016, marzo 10). Así funciona tu memoria. El País. https:// elpais.com/elpais/2016/02/22/ciencia/1456157540_110635.html

Martínez, G., Báez, E., Garza, J., Treviño, A. y Estrada, F. (2012). Implementación de un modelo de diseño curricular basado en competencias, en carreras de ingeniería. Innovación Educativa, 12(60), 87-103. https://www.redalyc.org/pdf/1794/179426856007.pdf

Moore, D. (2013). For interns, experience isn't always the best teacher. The Chronicle of Higher Education. https://www.chronicle.com/ article/For-Interns-Experience-Isnt/143073

Navarro, I., González, C., Álvarez, J., Fernández, F., y Heliz, J. (2014). Detección de dificultades de aprendizaje e implicación de las familias en la intervención. International Journal of Developmental and Educational Psychology, 7(1), 73-83. https://www. redalyc.org/pdf/3498/349851791008.pdf

Observatorio de Innovación Educativa del Tecnológico de Monterrey. (2015). Reporte Edutrends: Aprendizaje Basado en Retos. http://eduteka.icesi.edu.co/pdfdir/edutrends-aprendizaje-basado-en-retos.pdf

Ortíz, A. (2009). Aprendizaje y comportamiento basados en el funcionamiento del cerebro humano: emociones, procesos cognitivos, pensamientos e inteligencia. https://books.google.co.cr/ books?id=8md4zRdV2kwC\&printsec $=$ frontcover\& $\mathrm{dq}=$ apren- 
dizaje + por + emociones + ortiz\&hl $=$ en $\&$ sa $=X \& v e d=0 a h U K E w-$ jk0Ibnx9feAhWPr1kKHZJtAesQ6AEIKzAA\#v=onepage\&q$\& \mathrm{f}=$ false

Ortiz, D. (2015). El constructivismo como teoría y método de enseñanza. Sophia, Colección de Filosofía de la Educación, 19, 93-110. https://www.redalyc.org/pdf/4418/441846096005.pdf

Pérez, E. (2003). La pedagogía que vendrá: Más allá de la cultura escolar positivista. Utopía y Praxis Latinoamericana, 8(23), 87-95. https://www.redalyc.org/pdf/279/27982306.pdf

Poot-Delgado, C. (2013). Retos del aprendizaje basado en problemas. Enseñanza e Investigación en Psicología, 18(2), 307-314. http:// www.redalyc.org/pdf/292/29228336007.pdf

Robert, P. y Lisdero, P. (2016). Epistemología y metodología de la investigación sociológica: reflexiones críticas de nuestras prácticas de investigación. Sociologias, 18(41), 54-83. http://www.scielo. br/pdf/soc/v18n41/1517-4522-soc-18-41-00054.pdf

Román, C. (2012). ¿Qué hay detrás del buen rendimiento escolar en los estudiantes que ingresan a la universidad a través del Programa Propedéutico de la UCSH? Educere, 16(55), 331-338. https:// www.redalyc.org/pdf/356/35626140011.pdf

Saldarriaga, P., Bravo, G. y Loor, M. (2016). La teoría constructivista de Jean Piaget y su significación para la pedagogía contemporánea. Revista Cientifica Dominio de las Ciencias, 2(3), 127-137. https://dialnet.unirioja.es/descarga/articulo/5802932.pdf

Sánchez, J., Jiménez-Grajales, A., Sánchez, N. y González-Sepúlveda, J. (2013). Sinergia educativa: Adaptación de una Clase Magistral en un Instituto Tecnológico. Conciencia Tecnológica, 46, 17-23. https://www.redalyc.org/pdf/944/94429298004.pdf

Suárez, J., Maiz, F. y Meza, M. (2010). Inteligencias múltiples: una innovación pedagógica para potenciar el proceso enseñanza aprendizaje. Investigación y Postgrado, 25(1), 81-94. http://www.redalyc.org/comocitar.oa? $\mathrm{id}=65822264005$

Tünnerman, C. (2008). Modelos educativos y pedagógicos. Hispamer.

Viera, T. (2003). El aprendizaje verbal significativo de Ausubel. Algunas consideraciones desde el enfoque histórico cultural. Universidades, 26, 37-43 http://www.redalyc.org/pdf/373/37302605.pdf 